\title{
Is there a role of gut microbiota in mental health?
}

\author{
Klaus W. Lange ${ }^{\mathrm{a}^{*}}$, Katharina M. Lange ${ }^{\mathrm{a}}$, Yukiko Nakamura ${ }^{\mathrm{a}}$ \\ and Shigehiko Kanaya ${ }^{\mathrm{b}}$
}

\begin{abstract}
aDepartment of Experimental Psychology, University of Regensburg, Germany
${ }^{\mathrm{b}}$ Graduate School of Information Science, Nara Institute of Science and Technology, Japan

${ }^{*}$ Corresponding author: Klaus W. Lange, Institute of Psychology, University of Regensburg, 93040 Regensburg, Germany. Tel: +49 941 9433815; Fax: +49 941 9434496; E-mail: klaus.lange@ur.de
\end{abstract}

DOI: $10.31665 / J F B .2020 .9213$

Received: March 27, 2020; Revised received \& accepted: March 31, 2020

Citation: Lange, K.W., Lange, K.M., Nakamura, Y., and Kanaya, S. (2020). Is there a role of gut microbiota in mental health? J. Food Bioact. 9: 4-9.

\begin{abstract}
Research on the interaction between gut microbiota and the brain may have implications for our understanding of brain function, cognition, behavior and mental health. The literature on gut microbiota and its role in the pathophysiology and potential treatment of mental disorders has proliferated in recent years. Several neurodevelopmental disorders, including autism spectrum disorders, schizophrenia and attention-deficit/hyperactivity disorder, have been linked to the gut microbiota. The present perspective discusses the promise and pitfalls of gut microbiota research in relation to mental health. The manipulation of intestinal microbes in animals has revealed connections between gut microbiota and both normal and pathological brain functions. The hope fueling this research is that gut microbiota could be harnessed to prevent and treat mental disorders. The links observed between an imbalance of gut microbiota and impaired behavioral and mental states in humans are correlational. It is therefore essential to establish cause and effect relationships. No distinct gut microbiota patterns linked to different mental disorders have yet been identified. Large-scale, longitudinal trials need to examine whether the gut microbiota is a valid therapeutic target for mental disorders and whether pre-clinical findings and initial results of intervention trials (e.g., administration of probiotics) are of clinical relevance.
\end{abstract}

Keywords: Gut microbiota; Brain behavior research; Probiotics; Mental health; Psychiatry.

\section{Introduction}

The study of brain functions using neurophysiological, neuropsychological, neurochemical and neuroimaging techniques has long been the central approach to understanding the regulation of normal behavior as well as of altered behavioral states seen in mental disorders. However, emerging evidence suggests that researchers in this field have often neglected another important system involved in normal and abnormal brain functioning, the gastrointestinal system. This relationship seems to be an important one. The gut has a large, independent nervous system, and it communicates closely with the brain through the gut-brain axis. The human gut is colonized by bacteria, archaea and eukaryotes, the number of which has been estimated to be of the same order as the number of human cells in the body (Sender et al., 2016). The gut microbiota of humans is determined by genetic, epigenetic and dietary factors (Yadav et al., 2018). Early research studies in the 1980s began the mechanistical investigation of the influence of specific intestinal microorganisms on brain states in mammals (e.g., Brown et al., 1990). Findings of these studies revealed that gut microbes (microbiota) appear to affect a wide range of their host's physiological processes, including cell differentiation and proliferation, metabolic and immune functions, as well as behavior (Heiss and Olofsson, 2018; Nicholson et al., 2012; Rooks and Garrett, 2016; Sharon et al., 2016). Microbiota may therefore contribute to various diseases, such as inflammatory diseases, metabolic ailments, cancer and mental disorders. In particular, the gut microbiota plays 
an important role in modulating developmental processes of the central and enteric nervous systems and is involved in neurogenesis, myelination, glial cell function, synaptic pruning and bloodbrain barrier permeability (Heiss and Olofsson, 2019). Alterations in the bi-directional communication pathways between gut microbiota and brain may eventually lead to a disrupted neurotransmitter balance, increased chronic inflammation and heightened activity of the hypothalamic-pituitary-adrenal axis. Our current understanding of the complex communication systems involved in the gut microbiota-brain axis is limited.

\section{Gut microbiota in mental disorders}

Research on the interaction between complex communities of gut microbes (microbiota) and the brain may have implications for our understanding of brain function, cognition, behavior and mental health. Intestinal dysbiosis has been demonstrated to be linked to behavioral impairment (Bruce-Keller et al., 2015; Diaz Heijtz et al., 2011; Douglas-Escobar et al. 2013; Tillisch 2014), and this has given rise to investigations of the role of gut microbiota in mental health.

The findings of studies in germ-free mice, which lack intestinal and other microbiota, suggest that gut microbiota are essential for the development of neuronal systems involved in motor control, social activity and anxiety behavior (Desbonnet et al., 2014; Diaz Heijtz et al., 2011). Furthermore, exaggerated caution and disturbed social behavior displayed by germ-free BALB/c mice can be normalized by microbiome transplants from NIH Swiss mice, which do not show impaired exploratory and social behavior (Brinks et al., 2007). Conversely, NIH Swiss mice receiving $\mathrm{BALB} / \mathrm{c}$ microbiota transplants presented with marked hesitancy and caution (Bercik et al., 2011).

It has been demonstrated that neurodevelopmental mental disorders, including autism spectrum disorders (ASD), schizophrenia and attention-deficit/hyperactivity disorder (ADHD), are commonly associated with gastrointestinal symptoms, such as diarrhea and constipation (e.g., McElhanon et al., 2014; Severance et al., 2016). Furthermore, alterations in gut microbial composition have been observed in these conditions (Sharon et al., 2016), suggesting that microbial dysbiosis may be involved in their pathogenesis.

The role of gut microbiota has raised high expectations in mental disorders including the heterogeneous family of neurodevelopmental ASD, which are characterized by early-appearing deficits in cognitive, communicative and social skills and by repetitive sensory-motor behaviors (Lord et al., 2018). Children with ASD present with more gastrointestinal problems than healthy controls (McElhanon et al., 2014). Several dietary interventions, including gluten-free and casein-free diets (Lange et al., 2015; Reissmann et al., 2020) and omega-3 fatty acid supplementation (Lange, 2020a), have been suggested as complementary and alternative medicine treatments in ASD. However, in view of the less than convincing evidence in support of these dietary approaches, it would be premature to recommend them as alternatives in the management of ASD (Lange, 2020d; Reissmann et al., 2020).

ASD has been linked to an abnormal gut microbiota composition, with distinct increases or decreases in certain microbial groups (Mulle et al., 2013; O'Mahony et al., 2015). Elevated levels of Clostridia, Desulfovibrio (Finegold, 2011), Sutterella (Wang et al., 2013) and Bacteroidetes (Tomova et al., 2015) and decreased concentrations of Firmicutes, Prevotella, and Bifidobacter have been found in the stool of children with autism (Rosenfeld, 2015). However, other studies have found no compositional per- turbations of gut microbiota in individuals with ASD (Son et al., 2015). Moreover, alterations in gut microbial communities, which are frequently accompanied by strong food preferences, such as for starchy, processed foods, might be associated with behavioral problems in children with ASD (McElhanon et al., 2014). Reverse causation, i.e. behavioral changes leading to microbiota alterations, may explain the associations observed.

Schizophrenia is a complex, heterogeneous behavioral and cognitive syndrome, characterized by diverse symptoms, including cognitive dysfunction, positive symptoms, such as delusions and hallucinations, and negative symptoms, such as impaired motivation and social withdrawal (Owen et al., 2016). Few studies have investigated the microbiota composition in relation to schizophrenia. Individuals with first-episode psychosis have been reported to show increased numbers of Lactobacillus gut bacteria, which correlate with the severity of symptoms (Schwarz et al., 2017). In addition, a subgroup of these individuals, who showed the most profound alterations in microbiota composition, were less likely to respond to therapy (Schwarz et al., 2017). A potential involvement of the microbiota in schizophrenia has been suggested by findings showing that the administration of the antibiotic minocycline, which can induce changes in microbiota composition, potentiates the effect of the antipsychotic drug risperidone in people with chronic schizophrenia (Khodaie-Ardakani et al., 2014).

ADHD is a common psychiatric diagnosis in childhood and adolescence and is characterized by age-inappropriate levels of hyperactivity, inattention, and impulsivity. Individuals diagnosed with ADHD display long-term social, academic and mental health problems (Lange et al., 2010; Thapar and Cooper, 2016). While the etiology of ADHD appears to be multifactorial, definite causes remain unknown. Doubts regarding the effectiveness of commonly used ADHD therapies on clinically relevant outcome measures, the unproven long-term efficacy of treatment and concerns surrounding potentially serious adverse effects of medication have led to a search for alternative treatment options (Lange, 2017; Lange, 2018a; Lange, 2020b), including micro- and macronutrients and diets (Lange, 2018b; Lange, 2018c). Recent findings suggest that the gut microbiome dysbiosis associated with dietary patterns may be linked to a susceptibility to ADHD (Wang et al., 2020). However, a causal relationship has not been established.

To further our understanding of the effects of gut microbiota on mental disorders, future studies should also attempt to elucidate the mechanisms underlying the influence of microbiota on the health of the host. Potential mechanisms include direct activation of neuronal pathways (vagal afferents), microbial metabolism of nutrients and production of circulating mediators, and immune activation and circulating inflammatory mediators (Bruce-Keller et al., 2018).

\section{Therapeutic modulation of gut microbioata in mental disor- ders}

Tools of potential value in the modulation of gut microbiota as a treatment approach in mental disorders include fecal microbiota transplantation and dietary changes, such as the administration of probiotics. The success of microbiota transplantation in the treatment of refractory Clostridium difficile infection (Mattila et al., 2012) has excited some publicity. However, little information concerning the potential of this approach in mental disorders in humans is currently available. An open-label trial in children with autism has shown improved gastrointestinal and behavioral functions following fecal microbiota transplantation (Kang et al. 
2017). However, this trial was not randomized, placebo-controlled or blinded.

The composition of gut microbiota is determined in part by the diet. While an individual's gut microbiota is relatively stable over time, it responds quickly to dietary changes (David et al, 2014a, b). With respect to mental health, highly processed foods, which reduce intestinal microbial diversity, have been shown to be associated with an elevated risk of mental disorders (Dawson et al., 2016). Furthermore, several studies have revealed significant inverse associations between metrics of diet quality and disturbances of mental health (Flórez et al., 2015; Huddy et al., 2016; Mihrshahi et al., 2015; Rienks et al., 2013). However, the findings in regard to these relationships are mixed (Lai et al., 2016; Martínez-González and Sánchez-Villegas, 2016) and call for further controlled trials.

The administration of probiotics could potentially counterbalance the effects of Western diets on mental health. In one study, germ-free mice displayed exaggerated and maladaptive responses to stress, which were normalized by intestinal recolonization using probiotics (Sudo et al., 2004). Other studies have shown that probiotics improve mood, anxiety and cognition in animal models (Bravo et al., 2011; Desbonnet et al., 2010; Smith et al., 2014; Sudo et al., 2004). In humans, randomized trials have revealed beneficial effects of probiotics on mood (Messaoudi, 2011; Steenbergen, 2015). In children with autism, probiotics have been reported to be beneficial in normalizing the Bacteroidetes/Firmicutes balance and in elevating Desulfovibrio spp. levels (Tomova et al., 2015). More research is necessary to assess the amelioration of ASD symptoms following manipulation of the gut microbiota. A placebo-controlled trial has demonstrated that Lactobacillus casei reduces physiological responses to stress while increasing gut microbiota diversity (Kato-Kataoka, 2016). However, other studies found no benefits of probiotics versus placebo on mood, anxiety, stress or sleep quality in healthy volunteers (Kelly et al., 2017). In addition, the remarkable degree of inter-personal variability in human microbiota needs to be considered (Eckburg et al., 2005).

To sum up, the available evidence in support of the use of probiotics in the field of mental health is suggestive but not sufficient. Future trials evaluating probiotic use in various mental disorders are required in order to determine efficacy and to address several key problems, such as beneficial strains, dosage, method of administration and treatment duration (Wallace and Milev, 2017). Dietary fiber and prebiotics consistently modulate gut microbiota composition and function and could therefore become a valuable tool in research on the interaction between microbiota and brain (Dalile et al, 2019).

\section{Conclusions}

The experimental methods employed in seeking to establish relationships between gut microbes and behavior include alteration of the microbiota in animals and comparison of various behaviors of animals with and without microbiota. The manipulation of intestinal microbes has revealed connections between gut microbiota and both normal (emotion and cognition) and pathological (neurodevelopmental disorders, anxiety, mood) brain functions. The existing scientific literature has engendered hopes that gut microbiota could be harnessed in the prevention and treatment of mental disorders. If a direct relationship between gut dysbiosis and distinct psychopathological phenotypes could be established, this might lead to novel microbiome-based therapeutic strategies in psychiatry. However, the hype created by some researchers and the media seldom considers the pitfalls inherent in the interpretation of re- search findings in the field.

In humans, the links observed between an imbalance of gut microbiota and impaired behavioral and mental states are correlational. Therefore, it is essential to establish cause and effect relationships. Even if intestinal dysbiosis can causally facilitate mental disorders, the reciprocal effects of impaired brain neurophysiology on gut microbiota are unclear. Whether differences in gut microbiota cause disease or whether they are caused by the disease is often impossible to determine. Psychopathological symptoms and behavioral abnormalities may affect food preferences, for example, which in turn lead to gut dysbiosis. It is therefore important to ascertain whether gut dysbiosis is simply accompanying a disease or whether it is causing it.

Moreover, whether microbes mediate brain pathophysiology as well as mental health and disease, and, if so, whether individual or groups of different microbes are involved should be established. No distinct microbiota profile has as yet been related to any mental disorder. Since the exact alterations in gut microbiota underlying pathological changes are unknown even in animals, the diagnostic utility of microbiome analyses is currently limited in clinical practice. In addition, while there is some evidence suggesting in both animals and humans that exposure to transient microbes can influence brain physiology and behavior, there is no proof that changing the resident gut microbiota has therapeutic benefits. A detailed analysis of the interaction of the gut microbiota and nutritional modification at different neurodevelopmental stages may provide preventative strategies for neurodevelopmental disorders, including ASD, schizophrenia and ADHD.

An important aspect in relation to the difficulty in establishing distinct microbiological patterns concerns the phenotyping of psychiatric disorders. Given that the biological and environmental mechanisms involved in mental disorders are multifactorial, heterogeneous and complex, it is unrealistic to expect a one-fits-all solution in regard to gut microbiota. An examination of neuropsychiatric symptom dimensions may be of greater value than an investigation of specific disorders.

Five questions for researchers have been proposed as a measure to guard against hype in microbiome research (Hanage, 2014). These are: (1) can experiments detect differences that matter? (2) does the study show causation or just correlation? (3) what is the mechanism? (4) how much do experiments reflect reality? (5) could anything else explain the results? In respect of a possible role of gut microbiota in mental health, none of these questions can be answered in the affirmative: the experiments performed have been unable to detect differences that matter (no distinct microbiota patterns related to different mental disorders); the available studies show correlation but no causation (see above); the (animal) experiments do not reflect reality (germ-free mice etc.); the underlying mechanisms remain unknown; other factors could explain the results (e.g., food choices as a consequence of psychopathology.

In summary, gut microbiota-brain research has recently attracted a great deal of attention. Published research and review papers in the field have frequently presented misinterpretations of findings as well as speculation and overblown claims, which in turn have been propagated by the media. The interpretations of findings on the interactions between gut microbiota and behavior offer potential avenues for the treatment of mental disorders or even for the enhancement of normal cognition. However, the sobering news is that the radical claims made respecting causal relationships, in particular the extent to which the microbiota may control human behavior, are not justified. Pseudoscientific commercialization, especially claims by various companies that their analyses of individual fecal microbial content could form the basis of microbiome 


\section{Table 1. Gut microbiota and mental health}

The gut has a large, independent nervous system, which communicates bi-directionally with the brain through the gut-brain axis Connections between gut microbiota and both normal and pathological brain functions have been revealed

Gut microbiota affect a wide range of their host's physiological processes, including behavior

Gut microbiota are essential for the development of neuronal systems involved in social activity and anxiety behavior in rodents Intestinal dysbiosis is linked to behavioral impairment: Role of gut microbiota in mental health Highly processed foods, which reduce intestinal microbial diversity, are associated with an elevated risk of mental disorders

Altered gut microbial composition in neurodevelopmental disorders, including ASD, schizophrenia and ADHD, has been found: Involvement of microbial dysbiosis in their pathogenesis

The links observed between an imbalance of gut microbiota and behavioral and mental impairment in humans are correlational: Relationship of cause and effect? Reciprocal effects of impaired brain neurophysiology on gut microbiota

No distinct gut microbiota patterns have been linked to different mental disorders

Treatment approaches modulating gut microbiota in mental disorders include dietary changes (e.g. probiotics) and fecal microbiota transplantation

The potential of fecal microbiota transplantation in human mental disorders is unknown

Probiotics have been shown to improve mood, anxiety and cognition in animal models

Randomized trials have revealed beneficial effects of probiotics on mood in humans

Evidence in support of the use of probiotics in mental health is insufficient: beneficial strains, dosage, method of administration and treatment duration

Summary: Gut microbiota as a one-fits-all solution in mental health is unrealistic, since the pathogenesis of mental disorders is multifactorial and complex. Exact alterations in gut microbiota underlying mental disorders are unknown and microbiome analyses are of limited diagnostic use in clinical practice. Large-scale, longitudinal trials need to examine whether the gut microbiota is a valid therapeutic target in mental disorders.

cleansing or of personalized (mental) health interventions, are light on substance and heavy on salesmanship. Gut microbiota research can have no place in psychiatric practice until future large-scale, longitudinal trials have established whether the gut microbiota is a valid therapeutic target for mental disorders and whether the currently available pre-clinical findings and initial results of intervention trials are of clinical relevance (see Table 1).

\section{References}

Bercik, P., Denou, E., Collins, J., Jackson, W., Lu, J., Jury, J., Deng, Y., Blennerhassett, P., Macri, J., McCoy, K.D., Verdu, E.F., and Collins, S.M. (2011). The intestinal microbiota affect central levels of brain-derived neurotropic factor and behavior in mice. Gastroenterology 141: 599-609.

Bravo, J.A., Forsythe, P., Chew, M.V., Escaravage, E., Savignac, H.M., Dinan, T.G., Bienenstock, J., and Cryan, J.F. (2011). Ingestion of Lactobacillus strain regulates emotional behavior and central GABA receptor expression in a mouse via the vagus nerve. Proc. Nat. Acad. Sci. U. S. A. 108: 16050-16055.

Brinks, V., van der Mark, M., de Kloet, R., and Oitzl, M. (2007). Emotion and cognition in high and low stress sensitive mouse strains: A combined neuroendocrine and behavioral study in BALB/C and C57BL/6J mice. Front. Behav. Neurosci. 1: 8

Brown, R., Price, R.J., King, M.G., and Husband, A.J. (1990). Are antibiotic effects on sleep behavior in the rat due to modulation of gut bacteria? Physiol. Behav. 48: 561-565.

Bruce-Keller, A.J., Salbaum, J.M., and Berthoud, H.R. (2018). Harnessing gut microbes for mental health: Getting from here to there. Biol. Psychiatry 83: 214-223.

Bruce-Keller, A.J., Salbaum, J.M., Luo, M., Blanchard, E., Taylor, C.M., Welsh, D.A., and Berthoud, H.R. (2015). Obese-type gut microbiota induce neurobehavioral changes in the absence of obesity. Biol. Psychiatry 77: 607-615.

Dalile, B., Verbeke, K., Van Oudenhove, L., and Vervliet, B. (2019). Nourish- ing the gut microbiota: The potential of prebiotics in microbiotagutbrain axis research. Behav. Brain Sci. 42: e69.

David, L.A., Materna, A.C., Friedman, J., Campos-Baptista, M.I., Blackburn, M.C., Perrotta, A., Erdman, S.E., and Alm, E.J. (2014a). Host lifestyle affects human microbiota on daily timescales. Genome Biol. 15: R89.

David, L.A., Maurice, C.F., Carmody, R.N., Gootenberg, D.B., Button, J.E., Wolfe, B.E., Ling, A.V., Devlin, A.S., Varma, Y., Fischbach, M.A., Biddinger, S.B., Dutton, R.J., and Turnbaugh, P.J. (2014b). Diet rapidly and reproducibly alters the human gut microbiome. Nature 505: 559-563.

Dawson, S.L., Dash, S.R., and Jacka, F.N. (2016). The importance of diet and gut health to the treatment and prevention of mental disorders. Int. Rev. Neurobiol. 131: 325-346.

Desbonnet, L., Clarke, G., Shanahan, F., Dinan, T.G., and Cryan, J.F. (2014). Microbiota is essential for social development in the mouse. Mol. Psychiatry 19: 146-148.

Desbonnet, L., Garrett, L., Clarke, G., Kiely, B., Cryan, J.F., and Dinan, T.G. (2010). Effects of the probiotic Bifidobacterium infantis in the maternal separation model of depression. Neurosci. 170: 1179-1188.

Diaz Heijtz, R., Wang, S., Anuar, F., Qian, Y., Björkholm, B., Samuelsson, A., Hibberd, M.L., Forssberg, H., and Pettersson, S. (2011). Normal gut microbiota modulates brain development and behavior. Proc. Nat. Acad. Sci. U. S. A. 108: 3047-3052.

Douglas-Escobar, M., Elliott, E., and Neu, J. (2013). Effect of intestinal microbial ecology on the developing brain. JAMA Pediatr. 167: 374-379.

Eckburg, P.B., Bik, E.M., Bernstein, C.N., Purdom, E., Dethlefsen, L., Sargent, M., Gill, S.R., Nelson, K.E., and Relman, D.A. (2005). Diversity of the human intestinal microbial flora. Science 308: 1635-1638.

Finegold, S.M. (2011). Desulfovibrio species are potentially important in regressive autism. Med. Hypotheses 77: 270-274.

Flórez, K.R., Dubowitz, T., Ghosh-Dastidar, M.B., Beckman, R., and Collins, R.L. (2015). Associations between depressive symptomatology, diet, and body mass index among participants in the supplemental nutrition assistance program. J. Acad. Nutr. Diet 115: 1102-1108.

Hanage, W.P. (2014). Microbiome science needs a healthy dose of skepticism. Nature 512: 247-248.

Heiss, C.N., and Olofsson, L.E. (2019). The role of the gut microbiota in de- 
velopment, function and disorders of the central nervous system and the enteric nervous system. J. Neuroendocrinol. 31: e12684.

Heiss, C.N., and Olofsson, L.E. (2018). Gut microbiota-dependent modulation of energy metabolism. J. Innate Immun. 10: 163-171.

Hooks, K.B., Konsman, J.P., and O'Malley, M.A. (2019). Microbiota-gutbrain research: A critical analysis. Behav. Brain Sci. 42 e60: 1-53.

Huddy, R.L., Torres, S.J., Milte, C.M., McNaughton, S.A., Teychenne, M., and Campbell, K.J. (2016). Higher adherence to the Australian dietary guidelines is associated with better mental health status among Australian adult first-time mothers. J. Acad. Nutr. Diet 116: 1406-1412.

Kang, D.W., Adams, J.B., Gregory, A.C., Borody, T., Chittick, L., Fasano, A., Khoruts, A., Geis, E., Maldonado, J., McDonough-Means, S., Pollard, E.L., Roux, S., Sadowsky, M.J., Schwarzberg Lipson, K., Sullivan, M.B., Caporaso, J.G., and Krajmalnik-Brown, R. (2017). Microbiota Transfer Therapy alters gut ecosystem and improves gastrointestinal and autism symptoms: an open-label study. Microbiome 5: 10.

Kato-Kataoka, A. (2016). Fermented milk containing Lactobacillus casei strain Shirota preserves the diversity of the gut microbiota and relieves abdominal dysfunction in healthy medical students exposed to academic stress. Appl. Environ. Microbiol 82: 3649-3658.

Kelly, J.R., Allen, A.P., Temko, A., Hutch, W., Kennedy, P.J., Farid, N., Murphy, E., Boylan, G., Bienenstock, J., Cryan, J.F., Clarke, G., and Dinan, T.G. (2017). Lost in translation? The potential psychobiotic Lactobacillus rhamnosus (JB-1) fails to modulate stress or cognitive performance in healthy male subjects. Brain Behav. Immun. 61: 50-59.

Khodaie-Ardakani, M.R., Mirshafiee, O., Farokhnia, M., Tajdini, M., Hosseini, S.M., Modabbernia, A., Rezaei, F., Salehi, B., Yekehtaz, H., Ashrafi, M., Tabrizi, M., and Akhondzadeh, S. (2014). Minocycline add-on to risperidone for treatment of negative symptoms in patients with stable schizophrenia: randomized double-blind placebo-controlled study. Psychiatry Res. 215: 540-546.

Lai, J.S., Oldmeadow, C., Hure, A.J., McEvoy, M., Byles, J., and Attia, J. (2016). Longitudinal diet quality is not associated with depressive symptoms in a cohort of middle-aged Australian women. Br. J. Nutr. 115: 842-850.

Lange, K.W. (2017). The treatment of attention deficit hyperactivity disorder has no proven long-term benefits but possible adverse effects. Mov. Nutr. Health Dis. 1: 11-25.

Lange, K.W. (2018a). Lifestyle and attention deficit/hyperactivity disorder. Mov. Nutr. Health Dis. 2: 22-30.

Lange, K.W. (2018b). Do food bioactives play a role in attention-deficit/ hyperactivity disorder? J. Food Bioact. 4: 1-7.

Lange, K.W. (2018c). Diet, exercise, and mental disorders-public health challenges of the future. Mov. Nutr. Health Dis. 2: 39-59.

Lange, K.W. (2020a). Omega-3 fatty acids and mental health. Glob. Health J. $4: 18-30$.

Lange, K.W. (2020b). The need for alternative treatments for attentiondeficit/hyperactivity disorder. Mov. Nutr. Health Dis. 4: 1-8.

Lange, K.W. (2020c). Micronutrients and diets in the treatment of attention-deficit/hyperactivity disorder: Chances and pitfalls. Front. Psychiatry Child Adolesc. Psychiatry 11: 102.

Lange, K.W. (2020d). Lipids in the treatment of mental disorders. In: Shahidi, F. (Ed.). Bailey's Industrial Oil and Fat Products (7th ed). Wiley Blackwell.

Lange, K.W., Hauser, J., and Reissmann, A. (2015). Gluten-free and caseinfree diets in the therapy of autism. Curr. Opin. Clin. Nutr. Metab. Care 18: $572-575$.

Lange, K.W., Reichl, S., Lange, K.M., Tucha, L., and Tucha, O. (2010). The history of attention deficit hyperactivity disorder. Atten. Defic. Hyperact. Disord. 2: 241-255.

Lord, C., Elsabbagh, M., Baird, G., and Veenstra-Vanderweele, J. (2018). Autism spectrum disorder. Lancet 392: 508-520.

Martínez-González, M.A., and Sánchez-Villegas, A. (2016). Food patterns and the prevention of depression. Proc. Nutr. Soc. 75: 139-146.

Mattila, E., Uusitalo-Seppälä, R., Wuorela, M., Lehtola, L., Nurmi, H., Ristikankare, M., Moilanen, V., Salminen, K., Seppälä, M., Mattila, P.S. Anttila, V.J., and Arkkila, P. (2012). Fecal transplantation, through colonoscopy, is effective therapy for recurrent Clostridium difficile infection. Gastroenterology 142: 490-496.

McElhanon, B.O., McCracken, C., Karpen, S., and Sharp, W.G. (2014). Gastrointestinal symptoms in autism spectrum disorder: a meta-analy- sis. Pediatrics 133: 872-883.

Messaoudi, M. (2011). Assessment of psychotropic-like properties of a probiotic formulation (Lactobacillus helveticus R0052 and Bifidobacterium longum R0175) in rats and human subjects. Br. J. Nutr. 105: 755-764.

Mihrshahi, S., Dobson, A.J., and Mishra, G.D. (2015). Fruit and vegetable consumption and prevalence and incidence of depressive symptoms in mid-age women: results from the Australian longitudinal study on women's health. Eur. J. Clin. Nutr. 69: 585-591.

Mulle, J.G., Sharp, W.G., and Cubells, J.F. (2013). The gut microbiome: a new frontier in autism research. Curr. Psychiatry Rep. 15: 337.

Nicholson, J.K., Holmes, E., Kinross, J., Burcelin, R., Gibson, G., Jia, W., and Pettersson, S. (2012). Host-gut microbiota metabolic interactions. Science 336: 1262-1267.

O'Mahony, S.M., Stilling, R.M., Dinan, T.G., and Cryan, J.F. (2015). The microbiome and childhood diseases: Focus on brain-gut axis. Birth Defects Res. C 105: 296-313.

Owen, M.J., Sawa, A., and Mortensen, P.B. (2016). Schizophrenia. Lancet 388: 86-97.

Reissmann, A., Hauser, J., Stollberg, E., and Lange, K.W. (2020). Gluten-free and casein-free diets in the management of autism spectrum disorder: A systematic literature review. Mov. Nutr. Health Dis. 4: 21-38.

Rienks, J., Dobson, A.J., and Mishra, G.D. (2013). Mediterranean dietary pattern and prevalence and incidence of depressive symptoms in mid-aged women: results from a large community-based prospective study. Eur. J. Clin. Nutr. 67: 75-82.

Rooks, M.G., and Garrett, W.S. (2016). Gut microbiota, metabolites and host immunity. Nat. Rev. Immunol. 16: 341-352.

Rosenfeld, C.S. (2015). Microbiome disturbances and autism spectrum disorders. Drug Metab. Dispos. 43: 1557-1571.

Schwarz, E., Maukonen, J., Hyytiäinen, T., Kieseppä, T., Oresic, M., Sabunciyan, S., Mantere, O., Saarela, M., Yolken, R., and Suvisaari, J. (2017) Analysis of microbiota in first episode psychosis identifies preliminary associations with symptom severity and treatment response. Schizophrenia Res. 192: 398-403.

Sender, R., Fuchs, S., and Milo, R. (2016). Revised estimates for the number of human and bacteria cells in the body. PLoS Biol 14: e1002533.

Severance, E.G., Yolken, R.H., and Eaton, W.W. (2016). Autoimmune diseases, gastrointestinal disorders and the microbiome in schizophrenia: more than a gut feeling. Schizophr. Res. 176: 23-35.

Sharon, G., Sampson, T.R., Geschwind, D.H., and Mazmanian, S.K. (2016). The central nervous system and the gut microbiome. Cell 167: 915932.

Smith, C.J., Emge, J.R., Berzins, K., Lung, L., Khamishon, R., Shah, P., Rodrigues, D.M., Sousa, A.J., Reardon, C., Sherman, P.M., Barrett, K.E., and Gareau, M.G. (2014). Probiotics normalize the gutbrain-microbiota axis in immunodeficient mice. Am. J. Physiol. Gastrointest. Liver Physiol. 307: G793-802.

Son, J.S., Zheng, L.J., Rowehl, L.M., Tian, X., Zhang, Y., Zhu, W., Litcher-Kelly, L., Gadow, K.D., Gathungu, G., Robertson, C.E., Ir, D., Frank, D.N., and $\mathrm{Li}, \mathrm{E}$. (2015). Comparison of fecal microbiota in children with autism spectrum disorders and neurotypical siblings in the Simons Simplex Collection. PLoS One 10: e0137725.

Steenbergen, L. (2015). A randomized controlled trial to test the effect of multispecies probiotics on cognitive reactivity to sad mood. Brain Behav. Immun. 48: 258-264.

Sudo, N., Chida, Y., Aiba, Y., Sonoda, J., Oyama, N., Yu, X.N., Kubo, C., and Koga, Y. (2004). Postnatal microbial colonization programs the hypothalamic-pituitary-adrenal system for stress response in mice. J. Physiol. 558: 263-275.

Thapar, A., and Cooper, M. (2016). Attention deficit hyperactivity disorder. Lancet 387: 1240-1250.

Tillisch, K. (2014). The effects of gut microbiota on CNS function in humans. Gut Microbes 5: 404-410.

Tomova, A., Husarova, V., Lakatosova, S., Bakos, J., Vlkova, B., Babinska, K., and Ostatnikova, D. (2015). Gastrointestinal microbiota in children with autism in Slovakia. Physiol. Behav. 138: 179-187.

Wallace, C.J.K., and Milev, R. (2017). The effects of probiotics on depressive symptoms in humans: a systematic review. Ann. Gen. Psychiatry 16: 14.

Wang, L., Christophersen, C.T., Sorich, M.J., Gerber, J.P., Angley, M.T., and 
Conlon, M.A. (2013). Increased abundance of Sutterella spp. and Ruminococcus torques in feces of children with autism spectrum disorder. Mol. Autism 4: 42.

Wang, L.J., Yang, C.Y., Chou, W.J., Lee, M.J., Chou, M.C., Kuo, H.C., Yeh, Y.M., Lee, S.Y., Huang, L.H., and Li, S.C. (2020). Gut microbiota and dietary patterns in children with attention-deficit/hyperactivity disorder. Eur. Child Adolesc. Psychiatry 29: 287-297.

Yadav, M., Verma, M.K., and Chauhan, N.S. (2018). A review of metabolic potential of human gut microbiome in human nutrition. Arch. Microbiol. 200: 203-217. 